\title{
Neurosensory stimulation outdoors enhances cognition recovery in cognitive motor dissociation: A prospective crossover study
}

\author{
Caroline Attwell ${ }^{1, *}$, Jane Jöhr ${ }^{1}$, Alessandro Pincherle, Jean-Michel Pignat, Nina Kaufmann, \\ Jean-François Knebel, Loric Berney, Philippe Ryvlin and Karin Diserens \\ Department of Clinical Neurosciences, Neurology, Acute Neurorehabilitation Unit, University Hospital \\ CHUV, Lausanne, Vaud, Switzerland
}

\begin{abstract}
.
BACKGROUND: Neurosensory stimulation is effective in enhancing the recovery process of severely brain-injured patients with disorders of consciousness. Multisensory environments are found in nature, recognized as beneficial to many medical conditions. Recent advances detected covert cognition in patients behaviorally categorized as un- or minimally responsive; a state described as cognitive motor dissociation (CMD).

OBJECTIVE: To determine effectiveness of a neurosensory stimulation approach enhanced by outdoor therapy, in the early phases of recovery in patients presenting with CMD.

METHODS: A prospective non-randomized crossover study was performed. A two-phase neurosensory procedure combined identical individually goal assessed indoor and outdoor protocols. All sessions were video-recorded and observations rated offline. The frequency of volitional behavior was measured using a behavioral grid.

RESULTS: Fifteen patients participated in this study. The outdoor group patients had statistically significant higher number of intentional behaviors than the indoor group on seven features of the grid. Additionally, for all items assessed, total amount of behaviors in the outdoor condition where higher than those in the indoor condition.

CONCLUSIONS: Although preliminary, this study provides robust evidence supporting the effectiveness and appropriateness of an outdoor neurosensory intervention in patients with covert cognition, to improve adaptive goal-oriented behavior. This may be a step towards helping to restore functional interactive communication.
\end{abstract}

Keywords: Neurosensory stimulation, outdoor therapy, cognitive motor dissociation, covert cognition, early rehabilitation, disorders of consciousness

\section{Introduction}

Rehabilitation strategies for patients in the early phases of recovery after severe brain injury intend to improve attention and stimulate the networks

\footnotetext{
${ }^{1}$ These authors contributed equally to this work.

*Address for correspondence: Caroline Attwell, Department of Clinical Neurosciences, Neurology, Acute Neurorehabilitation Unit, University Hospital, Rue du Bugnon 46, Lausanne, Vaud, 1011 Switzerland. Tel.: +41 7955 67994; E-mail: caroline.attwell @ chuv.ch.
}

responsible for an individual to have conscious perception of himself and of his environment and adequately interact with it.

One type of intervention uses multisensory stimulation, which refers to a variety of methods used to stimulate the senses (sight, hearing, touch, taste and smell) that can considerably vary in form, intensity and number of modalities implied (Oh \& Seo, 2003). Multisensory environment is found in natural settings, which have been well recognized for serving as a resource for recovery and rehabilitation 
(Roger S Ulrich et al., 2008; Währborg, Petersson, \& Grahn, 2014).

In several conditions including neurological diseases, small but reliable evidence across studies supports nature-assisted therapies as an effective and appropriate intervention (see the review of (Annerstedt \& Wahrborg, 2011)). In a pioneering study, Ulrich (Ulrich, 1984) demonstrated that even simply viewing certain types of nature fosters improvements in clinical outcomes. Patients whose windows faced a park recovered faster after a surgery than patients whose windows faced a brick wall, in terms of reduced length of stay and pain medication. Similarly, Walch et al. (Walch et al., 2005) found that exposure to natural sunlight in post cervical and lumbar surgery patients, resulted in decreased stress, analgesic medication use, and pain medication costs. Individuals with Alzheimer's and dementia also benefit from spending time in outdoor environment, in terms of delay in cognitive decline (D'Andrea, Batavia, \& Sasson, 2007), stimulation of memory (Namazi \& Haynes, 1994), reduction of disruptive behaviors and sleep disturbances (Cohen-Mansfield \& Werner, 1998), or increase in levels of engagement and affect (Jarrott \& Gigliotti, 2010). In patients with neurological damage, Jonasson et al. (Jonasson, Marklund, \& Hildingh, 2007) qualitatively showed the capacity of gardens in improving their voluntary activity and functional ability.

Randomized control trials comparing groups receiving the usual care with a sensory stimulation program added are extremely scarce. Despite lack of scientific evidence (Lombardi, Taricco, De Tanti, Telaro, \& Liberati, 2002), it is hypothesized that applying a sensory stimulation program will enhance the recovery process and improve the outcomes of severely brain-injured patients experiencing an alteration in consciousness. Decades ago, LeWinn and Dimancescu (LeWinn \& Dimancescu, 1978) demonstrated greater improvement in sixteen comatose patients following multimodal sensory stimulation. Similarly, Mitchell (Mitchell, Bradley, Welch, \& Britton, 1990) showed that patients receiving a coma arousal procedure (described as a program of vigorous sensory stimulation) awakened from coma earlier than controls. A recent randomized controlled trial revealed significant improvements on validated scales dedicated to measure consciousness (Glasgow Coma Scale [GCS] and Western Neuro Sensory Stimulation profile [WNSSP]) after multimodal stimulation in comatose individuals after traumatic brain injury (Megha, Harpreet, \& Nayeem, 2013).
Disorders of consciousness often follow severe brain injuries and have been clinically defined according to behavioral criteria: the unarousable and unresponsive state of coma (Plum \& Posner, 1972), the unresponsive wakefulness syndrome (UWS) (Laureys et al., 2010), previously known as the vegetative state and the minimally conscious state (MCS) (Giacino et al., 2002). Unlike those in coma and UWS, patients in MCS show reproducible yet inconsistent intentional behavioral responses, revealing certain awareness of self and environment, indicators of conscious processing. Moreover, recent advances in functional imaging techniques have led to the identification of command-following during active/passive motor imagery tasks (i.e. covert cognition) in behaviorally unresponsive DOC patients (Edlow et al., 2017; Goldfine, Victor, Conte, Bardin, \& Schiff, 2011; Owen et al., 2006; Monti et al., 2010), supporting the operational definition of "cognitive motor dissociation" (CMD) proposed by Schiff (Schiff, 2015). A recent systematic review concluded that roughly $15 \%$ of UWS patients shows commandfollowing brain activity modulation (Kondziella, Friberg, Frokjaer, Fabricius, \& Moller, 2016).

A new behavioral assessment strategy, the Motor Behavior tool (MBT) developed by our group (Pignat et al., 2016) and recently revised and validated as a stand-alone form, MBT-r (Pincherle, 2019), enables the clinical identification of patients with behavioral signs of potential CMD. Indeed the MBT attempts to unveil subtle behavioral evidence of residual cognition, not detected by the neurobehavioral-validated scales.

To our knowledge, investigating whether sensory stimulation benefits patients presenting with CMD in the acute phase has not been so far explored. Here, we aim to determine the effectiveness of an interdisciplinary neurosensory program in the early phases of recovery in patients categorized as CMD with MBT in the context of the therapy outdoors. Furthermore, we will compare the neurosensory approach applied outdoors versus indoors.

\section{Materials and methods}

\subsection{Subjects}

Eligible patients enrolled in this non-randomized crossover study were adults in the acute phase of brain damage, admitted to the Acute NeuroRehabilitation Unit (NRA - Clinical Neurosciences 
Table 1

Clinical and demographic characteristics of patients

\begin{tabular}{|c|c|c|c|c|c|c|c|c|c|}
\hline & Age & Sex & Injury etiology & $\begin{array}{l}\text { Time } \\
\text { since } \\
\text { injury } \\
\text { (days) }\end{array}$ & $\begin{array}{l}\text { Initial clinical } \\
\text { diagnosis per } \\
\text { CRS-R }\end{array}$ & $\begin{array}{c}\text { MBT } \\
\text { classification }\end{array}$ & $\begin{array}{c}\mathrm{mRS} \text { at } \\
\text { admission }\end{array}$ & $\begin{array}{c}\mathrm{mRS} \text { at } \\
\text { discharge }\end{array}$ & $\begin{array}{c}\text { RoC } \\
(\mathrm{CRS}-\mathrm{R})\end{array}$ \\
\hline $\mathrm{P} 1$ & 51 & $\mathrm{~F}$ & IS & 19 & MCS & CMD & 5 & 4 & Yes \\
\hline $\mathrm{P} 2$ & 53 & $\mathrm{~F}$ & $\begin{array}{l}\text { Metabolic } \\
\text { encephalopathy }\end{array}$ & 49 & UWS & CMD & 5 & 4 & Yes \\
\hline P3 & 61 & M & $\mathrm{IH}$ & 15 & COMA & CMD & 5 & 4 & No \\
\hline P4 & 60 & M & $\mathrm{IH}$ & 26 & COMA & CMD & 5 & 4 & Yes \\
\hline P5 & 78 & M & $\begin{array}{l}\text { Infectious } \\
\text { encephalopathy }\end{array}$ & 48 & COMA & CMD & 5 & 4 & No \\
\hline P6 & 43 & M & TBI & 19 & COMA & CMD & 5 & 3 & Yes \\
\hline P7 & 60 & $\mathrm{~F}$ & $\mathrm{SAH}$ & 19 & COMA & CMD & 5 & 5 & No \\
\hline P8 & 68 & $\mathrm{~F}$ & $\begin{array}{l}\text { Multifactorial } \\
\text { encephalopathy }\end{array}$ & 36 & MCS & CMD & 5 & 5 & No \\
\hline P9 & 53 & $\mathrm{~F}$ & TBI & 21 & COMA & NON CMD/DOC & 5 & 5 & No \\
\hline P10 & 50 & M & $\begin{array}{l}\text { Anoxic } \\
\text { encephalopathy }\end{array}$ & 22 & MCS & $\mathrm{CMD}$ & 5 & 3 & Yes \\
\hline P11 & 59 & $\mathrm{~F}$ & $\mathrm{IH}$ & 26 & MCS & CMD & 5 & 4 & Yes \\
\hline $\mathrm{P} 12$ & 78 & $\mathrm{~F}$ & $\mathrm{SAH}$ & 26 & MCS & CMD & 5 & 5 & No \\
\hline P13 & 27 & M & TBI & 18 & UWS & CMD & 5 & 2 & Yes \\
\hline P14 & 25 & $\mathrm{~F}$ & TBI & 22 & UWS & CMD & 5 & 4 & Yes \\
\hline $\mathrm{P} 15$ & 23 & M & TBI & 12 & COMA & CMD & 5 & 3 & Yes \\
\hline
\end{tabular}

IS = Ischemic stroke; IH = Intraparenchymal hemorrhage; $\mathrm{SAH}=$ subarachnoid hemorrhage; TBI = Traumatic brain injury; CRS-R = Coma Recovery Scale-Revised; MCS = minimally conscious state; UWS = unresponsive wakefulness syndrome; MBT = Motor Behaviour Tool; $\mathrm{CMD}=$ Cognitive-motor dissociation; $\mathrm{mRS}=$ Modified Rankin Scale $; \mathrm{RoC}=$ Recovery of consciousness.

Department, University Hospital of Lausanne, Switzerland) between May 2015 and July 2017. Before admission to the NRA, the patients in the intensive care unit were diagnosed by clinical neurologists and neuropsychologists after repeated testing using the Coma Recovery Scale-Revised (CRS-R) and Motor-Behavior Tool (MBT). Clinical details and diagnoses are shown in Table 1. All included patients were clinically stable for transport to a therapeutic garden and had no major medical problems interfering with their safety while outdoors. Eligible patients were screened for the inclusion and exclusion criteria by a neurologist. The experimental protocol (142/09) was approved by the Local Lausanne Ethics Committee and written informed consent was provided by the patients' legal authorized representatives. All included patients underwent a daily 300-minute interdisciplinary program of rehabilitation according to their clinical condition. We used the modified Rankin Scale (mRS) as a clinical outcome measure on NRA admission and on hospital discharge.

\subsection{Procedure}

The entire study population followed the same protocol with specified activities adapted to each patient's abilities. Tasks were individually identi- fied to suit the patient resulting in an individual goal assessment (SMART goals, see (Bovend'Eerdt, Botell, \& Wade, 2009)). Moreover, levels were individually set following the shaping method of learning, which allows to approach a desired motor or behavioral objective in small steps of increasing difficulty or increasing demand (Taub et al., 1994).

We designed a two-phase procedure combining an identical individually goal assessed protocol carried out indoors and outdoors. Average length of protocol sessions was 25 minutes (initial rest and therapy). The protocol order was randomized and both sessions were applied the same day by the same therapist reproducing indoors and outdoors the therapy protocol designed for the patient. Maximum time in between the two sessions (including length of sessions) was 5 hours.

\subsection{Interventions}

All indoor sessions were conducted inside the hospital, either in the intermediate care unit, or in a dedicated physiotherapeutic area. All outdoor sessions occurred in the therapeutic garden located in the enclosure of the hospital. This enriched outdoor setting was only used by patients and was specifi- 
cally designed to allow accessibility and safety for patients with mobility difficulties. Its design focused on increasing multisensory stimulation by providing various structured and adapted spaces stimulating the senses (i.e., variety of plants and trees, planted aromatic herbs, fountain), as well as several therapeutic training spots (i.e., sensory walking path, parallel bars, stairs, espalier, soft and hard ground).

In the initial 5 to 10 minutes rest period, patients were assessed for pain using the Critical Care Pain Observation Tool (CPOT) (Gelinas, Fortier, Viens, Fillion, \& Puntillo, 2004) or the Visual Analog Scale (Bijur, Silver, \& Gallagher, 2001). The CPOT is designed to score the pain of patients who are not able to report it themselves while the VAS is designed for patients who can. Pain was assessed to ensure the comfort and safety of the subjects during the trial.

Then, patients underwent either physiotherapy and/or occupational therapy; treatment previously chosen according to the patient's abilities and always aimed at improving the voluntary and/or purposeful behavior of the patient.

All sessions were video-recorded for offline analyses. Two independent blinded and trained investigators reviewed all videos and rated all voluntary and/or purposeful behavior and initiation performance using the behavioral grid described below.

Usual environment effects (e.g. noises, staff, visitors, alarms) were not attenuated but served as background stimulation.

\subsection{Measurements}

Voluntary and/or purposeful behavior were measured by a behavioral grid (see supplementary material), developed by the NRA research team and primarily based on the overall structure of the CRSR scale (Kalmar \& Giacino, 2005). The grid allows objective behavior counting and prevents a potential training effect as it is observational and repetition of specific behaviors is not required.

This tool consists of 44 items comprising six sub-categories addressing auditory, visual, motor, oro-motor, communication and attention functions. Counting is based on the presence or absence of a defined behavioral response to specific sensory stimuli. The presence and total numbers of responses (frequency) were counted, as frequency is an indicator of voluntary and/or purposeful behavior, index of a conscious perception. All observed behaviors were taken into account without resulting in any cutoff or classification. The rated observations were separated into two condition groups (indoor and outdoor) to allow further analyses. The content of the instrument was internally assessed by a panel of recognized experts representing all neurorehabilitation disciplines (neurology, speech therapy, physiotherapy, occupational therapy and neuropsychology).

\subsection{Statistical analysis}

The inter-rater agreement of the behavioral grid was measured for each clinical item. Cohen's kappa coefficient $(\kappa)$ was used to measure inter-rater agreement; acceptable inter-rater agreement was set at $\kappa>0.7$.

Analyses were performed using MATLAB 12.0 (Math-Works, Natick, Massachusetts, USA). This study used a two-way repeated measure Anova design $(2 \times 2)$ with two within-subject factors, expert (expert 1 vs expert 2) and condition group (difference in/out vs zero). Given the size of each group (indoor and outdoor), and the non-normality of the groups based on a preliminary Lilliefors test, nonparametric statistics were used throughout. The nonparametric two-way repeated measure ANOVA was performed using bootstrappin (Knebel, Javitt, \& Murray, 2011). The subject label was bootstrapped and the within subject condition was permuted within the subject. This approach keeps the intra-subject variance. On each cycle, we calculated the F-values for each randomization. Repeating this for 1000 cycles generates an empirical distribution of $F$-values from which a corresponding $p$-value can be obtained by comparison to the original $F$-values. The only hypothesis is that our data represent the space that we would like to test.

In addition, to avoid multiple testing problems the $p$-value was adapted using Bonferroni correction.

\section{Results}

The behavioral grid instrument has strong inter-rater reliability $(\kappa=0.9385)$. Clinical and demographic characteristics of patients are listed in Table 1. Twenty participants were enrolled in the study. Five patients were excluded from the final sample (one patient withdrew consent, three patients did not undergo the complete intervention and one patient died). Of the included patients, 11 were males and 4 were females with an overall mean age $52.6 \pm 17.2$ years. The average time from injury was $25.2 \pm 10.9$ days. Etiologies differed; five (33.3\%) suffered 
Table 2

Mean difference between the indoor and outdoor protocols regarding total number of behavioral responses

\begin{tabular}{|c|c|c|c|c|}
\hline $\begin{array}{l}\text { Function } \\
\text { sub-categories }\end{array}$ & Behavioral Responses & Mean & SD & $\begin{array}{c}\text { Multiple } \\
\text { correction } \\
p \text {-value }\end{array}$ \\
\hline \multirow[t]{8}{*}{ Auditory } & Reaction to a simple command & 2.13 & 4.18 & $<0.001 * * *$ \\
\hline & Reaction to a semi-complex command & 0.08 & 0.51 & 1.711 \\
\hline & Reaction to a complex command & 0.00 & 0.00 & 4.74 \\
\hline & Head orients toward an auditory stimulus & 0.47 & 2.42 & 0.35 \\
\hline & Eyes orient toward an auditory stimulus & 0.56 & 2.42 & $<0.005^{* *}$ \\
\hline & Eyes opening in response to an auditory stimulus & 0.03 & 0.52 & 5.52 \\
\hline & Eyes opening on command & 0.05 & 0.56 & 4.424 \\
\hline & Reaction when addressed by own name & 0.34 & 1.28 & $<0.001 * * *$ \\
\hline \multirow[t]{8}{*}{ Visual } & Spontaneous exploration/fixation of an object & 0.44 & 3.25 & 2.304 \\
\hline & Exploration/fixation of an object on command & 0.48 & 3.28 & 1.792 \\
\hline & Visual pursuit of an object on command & 0.16 & 0.90 & 0.296 \\
\hline & Spontaneous exploration/fixation of an individual (person) & 0.67 & 4.27 & 1.333 \\
\hline & Exploration/fixation of an individual on command & 0.74 & 2.44 & $<0.001^{* * *}$ \\
\hline & Spontaneous visual pursuit of a moving object or individual & 1.01 & 5.94 & 0.594 \\
\hline & Pursuit of an individual on command & 0.02 & 0.15 & 2.369 \\
\hline & Spontaneous designation of an object & 0.00 & 0.00 & 4 \\
\hline \multirow[t]{8}{*}{ Motor } & Designation of an object on command & 0.00 & 0.00 & 3 \\
\hline & Choice of an object on command & 0.01 & 0.11 & 5.088 \\
\hline & Spontaneous seizing of an object & 0.30 & 1.59 & 0.408 \\
\hline & Seizing of an object on command & 0.29 & 1.68 & 0.992 \\
\hline & Spontaneous movement related to an object/task/individual & 1.90 & 4.34 & $<0.001 * * *$ \\
\hline & Movement on command & 2.38 & 6.10 & $<0.001 * * *$ \\
\hline & Imitation of a movement & 0.20 & 1.44 & 2.052 \\
\hline & Eyes open on tactile stimulus & 0.07 & 0.91 & 4.667 \\
\hline \multirow[t]{7}{*}{ Oro-motor } & Teeth grinding & 0.01 & 0.08 & 3.135 \\
\hline & Yawning & 0.26 & 1.27 & 0.324 \\
\hline & Sighing & 0.37 & 2.77 & 2.225 \\
\hline & Smiling/laughing & 0.13 & 0.83 & 1.333 \\
\hline & Frowning/gesturing & 0.01 & 1.37 & $\mathbf{5 . 0 8 2}$ \\
\hline & Spontaneous swallowing & 0.56 & 1.56 & $<0.001 * * *$ \\
\hline & Swallowing on command & 0.02 & 0.61 & 5.184 \\
\hline \multirow[t]{9}{*}{ Communication } & Response following an established communication code & 0.26 & 2.11 & 2.331 \\
\hline & Spontaneous audible vocalization & 0.05 & 0.67 & 5.088 \\
\hline & Spontaneous audible production of a word & 0.17 & 1.36 & 2.369 \\
\hline & Spontaneous audible production of a simple or complex sentence & 0.04 & 2.05 & 5.082 \\
\hline & Audible vocalization on command & 0.12 & 1.32 & 4.641 \\
\hline & Audible production of a word on command & 0.23 & 2.68 & 4.624 \\
\hline & Audible production of a simple or complex sentence on command & 0.11 & 2.90 & 5.256 \\
\hline & Non-audible diction & 0.23 & 2.10 & 3 \\
\hline & Raising the voice & 0.02 & 0.15 & 2.052 \\
\hline \multirow[t]{4}{*}{ Attention } & Attention on a stimulus held for 5 secs, at least $3 \&$ times during the whole activity & 0.01 & 0.11 & 5.52 \\
\hline & Distracting stimulus self-inhibited & 0.01 & 0.08 & 2 \\
\hline & Distracting stimulus ( $\max 10 \mathrm{secs}$ ) and resuming the task without external stimulation & 0.01 & 0.08 & 4.806 \\
\hline & Distracting stimulus ( $\max 10 \mathrm{secs}$ ) and resuming the task with external stimulation & 0.02 & 0.17 & 4.806 \\
\hline
\end{tabular}

Results are presented as mean \pm standard deviation of the difference in the number of behavioral responses between outdoor and indoor conditions; ${ }^{* *}$ significant at $p<0.005 ;{ }^{* * *}$ significant at $p<0.001$.

a trauma, four various encephalopathies (26.7\%), three an intraparenchymal hemorrhage (20\%), two a subarachnoid hemorrhage (13.3\%), and one an ischemic stroke $(6.6 \%)$. Initial clinical diagnosis using the CRS-R classified five patients as MCS, three UWS and seven comatose. All but one of the patients were categorized as in CMD state, using the MBT. All included subjects completed at least three sessions of the protocol. On NRA admission, all patients had
$\mathrm{mRS}$ scores of 5 (severe disability). On hospital discharge, mRS scores ranged from 2 (slight disability) to 5 with a mean of 4 (moderately severe disability). Nine patients recovered consciousness according to the final CRS-R evaluation (defined by functional use of objects and/or functional communication) on discharge,

A summary of the results of the behavior measures is shown in Table 2. 
Outdoor group sessions had a significantly greater frequency of behaviors than the indoor group sessions on seven items of the grid. During intervention, the sum of counted behaviors was significantly larger in the outdoor condition for specific items of the auditory, visual, motor and oro-motor function sub-categories. Additionally, for all items assessed, total amount of behaviors in the outdoor condition where higher than those in the indoor condition.

Amongst the eight auditory responses, three of them were observed more frequently outside including, "reaction to a simple command" $(p<0.001)$, "eyes oriented toward an auditory stimulus" $(p<0.005)$ and "reaction when addressed by own name" $(p<0.001)$.

On the other hand, only one of the eight visualbehavior items, "exploration/fixation of an individual on command" ( $p<0.001)$ was more frequent in the open-air.

Two of the eight motor "spontaneous movement related to an object/task/individual" $(p<0.001)$ and "movement on command" $(p<0.001)$ and one of the seven oro-motor components, "swallowing" $(p<0.001)$ were more frequently observed during outdoor therapy.

The highest frequencies of activity in the outdoor condition was observed for the "movement on command" item of the motor sub-categories (mean difference $=2.38$ ).

We observed no statistically significant differences in items of communication and attention sub-categories between the outdoor and indoor conditions.

We found no evidence of unintended effects in any subject, including no differences in mean VAS/CPOT scores between conditions.

\section{Discussion}

The purpose of the study was to evaluate the effectiveness of outdoor interdisciplinary neurosensory approach for improving the recovery of volitional behaviors and interaction abilities in patients presenting with CMD. To our knowledge, this is the first study to investigate the effect of this type of intervention on this group of patients.

Overall, the results show a significant effect of the therapies outdoors compared to therapies indoors with a higher number of observed voluntary purposeful behaviors and movement initiation when stimulated outdoors.
The improvement after neurosensorial stimulation in an open-air environment may be attributed to the well-recognized and documented restorative effects of nature on cognitive, emotional and physical functioning (for a review see, (Maller, Townsend, Pryor, Brown, \& St Leger, 2006)). Integrative frameworks such as the «biophilia hypothesis » by Wilson (Wilson, 1984), postulating that humans have a genetically based need to interact with nature; or the « concept of therapeutic landscapes »(Gesler, 1992) focusing on everyday landscapes believed to contribute to healing, both endorse the therapeutic benefit that exposure to nature may have on human health.

The effectiveness found in the present study can also be explained based on the " attention restoration theory » (ART) proposed by Kaplan \& Kaplan (Kaplan \& Kaplan, 1989) which suggests that nature can restore the attentional capacity. This theory postulated two components of attention: the non-demanding « involuntary attention » passively stimulated by sensory stimuli (i.e., colors, motion, contrasts, sounds); and the restricted « directed attention » requiring a voluntary engagement directed by executive function processes, ultimately resulting in mental fatigue. Exposure to direct or indirect inferences to nature engages the involuntary, sparing the directed, thus helping the directed attention capacities to recover. Empirical evidence of ART is mostly descriptive and based on observations of humannature interactions and analysis of quantitative data. Nonetheless, a recent systematic review supported the impact of exposure to nature environments on attention, though concluded about the uncertainty regarding which aspects of attention may be affected (Ohly et al., 2016).

The coma following brain injury produces sensory deprivation (Ansell, 1991), thus in order to prevent such additional detrimental effects on the already damaged brain, the rationale for treatment leads to enrich the environment and promote neural plasticity (Di \& Schnakers, 2018). Interventional approaches resort to variations of multisensory stimulation such as multimodal stimulation of the senses (Canedo, Grix, \& Nicoletti, 2002), music therapy (Formisano et al., 2001; Magee, 2007), or verticalization protocol using a tilted table with an integrated stepping device (Krewer, Luther, Koenig, \& Muller, 2015; Frazzitta et al., 2016). Our findings are consistent with those reported in these studies attesting improvements on the degree of arousal, the interaction with the environment, the psychomotor initiative or the level of 
consciousness. To some extent, our results confirm the previous findings of Lanz et al. (Lanz, Moret, Rouiller, \& Loquet, 2013) regarding multisensory integration in non-human primates. In their study subjects were engaged in a detection sensory-motor task where visual and auditory stimuli were displayed individually or simultaneously. Mainly, they demonstrated a multisensory advantage increasing speed of stimulus detection and improving performance accuracy: motor responses were better and faster when subjects were exposed to multimodal sensory stimulation.

Furthermore, the significant improvements demonstrated in seven items of our behavioral grid concern four of the main modalities assessed by the CRS-R, which is the validated and recommended scale for use in individuals with disorders of consciousness (American Congress of Rehabilitation Medicine et al., 2010). One interesting point is that six of these items involve signs of volitional top-down cognition implying that the patient understood the verbal command and willfully responded, therefore attesting level of conscious awareness. First, this endorses the initial assumption made on the MBT assessment (i.e. being in a CMD condition), identifying these patients as presenting subtle motor behaviors consistent with residual cognition. Besides, nine CMD patients behaviorally demonstrated consciousness recovery at discharge, as assessed by the CRS-R scoring signs qualifying emergence, hence supporting the correlation between the presence of residual cognition at an early stage and subsequent consciousness recovery (Pignat et al., 2016); (Pincherle, 2019). Second, this finding is in line to a certain extent with those reported in studies exploring the detection of covert awareness in patients with DOC by means of complementary neuroimaging approaches. Indeed, signs of such covert cognition can be detected in roughly $15 \%$ of patients in a behavioral state of UWS with functional MRI and EEG command-following paradigms (e.g. (Monti et al., 2010; Cruse et al., 2011; Edlow et al., 2017). Recently, Curley et al. (Curley, Forgacs, Voss, Conte, \& Schiff, 2018) even demonstrated a higher rate of responders to command (13/20 patients with severe DOC studied) using a new approach at the bedside with repetitive testing with EEG command-following protocols.

It should also be noted that no significant improvement was observed for the rest of the items of the grid, where most of them implied cognitive top- down processes compatible with the condition of CMD patients. It could be argued that their graded higher complexity requiring multiple actions (i.e., semi-complex and complex orders), or the integration of another stimulus (i.e., object, person), may hamper the execution of the measured behaviors, in view of concomitant neuropsychological deficits expected in such brain-injured patients. This result (or lack of) might simply reflect an estimate of the level of cognitive performance of the CMD patient.

Motivational drive mechanisms could easily be considered to explain the increase in the frequency of volitional behaviors observed in the present study. Motivation impacts behavior and has received much concern in the literature, yet the exact mechanisms underlying its neurophysiology remain unclear. In the case of the most severe disorder of motivation, i.e. akinetic mutism syndrome (AM), characterized by profound apathy and a lack of verbal and motor output for action, despite preserved alertness and the intention to speak, meaningful responses can be occasionally elicited when acting on mesocortical dopamine pathways (Spiegel, Casella, Callender, \& Dhadwal, 2008). In the so-called «telephone effect », patients with AM appear to speak better whilst on the phone; a phenomenon explained by a temporary reversal in the severe decrease in drive by a release of dopamine which activates the damaged motivational circuit and results in brief verbalizations (Yarns \& Quinn, 2013). The clinical presentation of the patients in our study resemble those of AM in a less severe intensity, therefore it is conceivable that the relevance brought by an outdoor environment may modulate and increase the activation of such dopaminergic circuits and improve the realization of the action itself.

This hypothesis also finds support in the approach developed by Bosch-Bouju et al. (Bosch-Bouju, Hyland, \& Parr-Brownlie, 2013) who emphasize the role of multiple driver or driver-like inputs in motor control, including a strong influence of motivational mechanisms. They proposed that, in order to evoke an optimal movement, the motor thalamus acts more than a simple relay structure, but as a « superintegrator » of information from the prefrontal cortex (initiating the motor program), the basal ganglia (processing motivational information) and the cerebellum (processing complex proprioceptive information), which then sends all super-integrated signals back to the cortex. According to this view, we can speculate that promoting motivational stimulation and increas- 
ing sensory inputs may modulate neural activity of the loop, resulting in a better generation of motor programs. This interpretation is supported by the fact that there were no measured behaviors more numerous in the indoor condition, considered as less motivational and with lower multisensory stimulation.

Stimuli with emotional salience also provide a reliable motivational resource. Emotional stimuli capture attention (Phelps, 2006), are prioritized in cognitive system, or intensify sensory integration (Vuilleumier, 2005). Although emotional stimuli were not directly provided here, a significant effect was found when addressing the patient with her or his own name. This finding is consistent with those reported in several studies with DOC patients that use the patient's name as an effective stimulus. For instance, name sequences containing the patient's own name or other names were presented to 8 UWS and 14 MCS in passive and active (instructed to count their own name) conditions of an evoked-related potentials paradigm (Schnakers et al., 2008). Results demonstrated that MCS patients, like controls, presented a greater P3 (the third positive wave of event-related potentials) to their own name in both conditions. Further research must be conducted to reach more concrete conclusions regarding the benefits of emotional salience on increasing volitional behaviors in patients presenting with CMD.

Another limitation of our study concerns the absence of randomization, due to obvious ethical considerations, as well as the difficulty to ascertain the degree to which the benefits of the study interventions are independent from the effect of the individualized rehabilitation program followed by each patient. However, the overall amount and intensity of rehabilitative therapies were controlled and did not differ significantly between patients (patients whose clinical conditions require a less intensive rehabilitation program were not included in the study). Furthermore, it is not known whether being outdoors affects the intervention of the therapists in terms of their mood improvement or increased motivation to stimulate the patient, which may have influenced response to the intervention.

Further studies may use additional measurements to evaluate more specifically motivational aspects such as participation performances (by patients and by therapists) or reactions to specific emotionally salient stimuli. In addition, physiological measurements such as salivary cortisol to evaluate the effect of natural settings on the stress level of patients with severe brain injuries would be valuable.

\section{Conclusions}

Our findings suggest that outdoor therapy provides a beneficial complementary rehabilitative treatment for patients in the early phases following a severe brain injury. Although preliminary, this study shows robust evidence supporting the effectiveness and appropriateness of an outdoor neurosensory intervention in disorders of consciousness, especially for patients presenting with CMD. The outdoor approach improves adaptive goal-oriented behaviors hence helping to restore a functional interactive communication. Notwithstanding the small sample size, the results are promising and demonstrate their applicability even with a heterogeneous etiology, as well as their easiness to apply to any health-care setting that has access to outdoors facilities. Finally, in a more global perspective, they advocate for the restorative benefits of natural settings as a relevant resource for public health.

\section{Acknowledgments}

The authors would like to thank the families of the patients for giving their consent to our study. Thanks to the therapists for performing the therapeutic interventions, to Veronique de Goumoëns for her investment and senior advice, to Krystel Bruyère for her methodological input, to Hélène Brioschi Levi for her support and to Melanie Hirt for English-language editing and proofreading the manuscript.

\section{Conflict of interest}

The authors declare no conflicts of interest with respect to the authorship and/or publication of this article. This study was partially supported by a grant from The Gianni Biaggi de Blasys Foundation.

\section{References}

American Congress of Rehabilitation Medicine, B. I.-I. S. I. G. D. o. C. T. F., Seel, R. T., Sherer, M., Whyte, J., Katz, D. I., Giacino, J. T.,... \& Zasler, N. (2010). Assessment scales for disorders of consciousness: Evidence-based recommendations for clinical practice and research. Arch Phys Med Rehabil, 91(12), 1795-1813. doi:10.1016/j.apmr.2010. 07.218

Annerstedt, M., \& Wahrborg, P. (2011). Nature-assisted therapy: Systematic review of controlled and observa- 
tional studies. Scand J Public Health, 39(4), 371-388. doi: $10.1177 / 1403494810396400$

Ansell, B. J. (1991). Slow-to-recover brain-injured patients: Rationale for treatment. J Speech Hear Res, 34(5), 1017-1022.

Bijur, P. E., Silver, W., \& Gallagher, E. J. (2001). Reliability of the visual analog scale for measurement of acute pain. Acad Emerg Med, 8(12), 1153-1157.

Bosch-Bouju, C., Hyland, B. I., \& Parr-Brownlie, L. C. (2013). Motor thalamus integration of cortical, cerebellar and basal ganglia information: Implications for normal and parkinsonian conditions. Front Comput Neurosci, 7, 163. doi:10.3389/fncom.2013.00163

Bovend'Eerdt, T. J., Botell, R. E., \& Wade, D. T. (2009). Writing SMART rehabilitation goals and achieving goal attainment scaling: A practical guide. Clinical Rehabilitation, 23(4), 352361.

Canedo, A., Grix, M. C., \& Nicoletti, J. (2002). An analysis of assessment instruments for the minimally responsive patient (MRP): Clinical observations. Brain Inj, 16(5), 453461. doi:10.1080/02699050110119853

Cohen-Mansfield, J., \& Werner, P. (1998). The effects of an enhanced environment on nursing home residents who pace. The Gerontologist, 38(2), 199-208.

Cruse, D., Chennu, S., Chatelle, C., Bekinschtein, T. A., Fernandez-Espejo, D., Pickard, J. D.,... Owen, A. M. (2011). Bedside detection of awareness in the vegetative state: A cohort study. Lancet, 378(9809), 2088-2094. doi:10.1016/S01406736(11)61224-5

Curley, W. H., Forgacs, P. B., Voss, H. U., Conte, M. M., \& Schiff, N. D. (2018). Characterization of EEG signals revealing covert cognition in the injured brain. Brain, 141(5), 1404-1421. doi:10.1093/brain/awy070

D’Andrea, S. J., Batavia, M., \& Sasson, N. (2007). Effect of Horticultural Therapy on Preventing the Decline of Mental Abilities of Patients with Alzheimer's Type Dementia. Journal of Therapeutic Horticulture, 18.

Di, H., \& Schnakers, C. (2018). Sensory stimulation program. In Coma and Disorders of Consciousness (pp. 169-179): Springer.

Edlow, B. L., Chatelle, C., Spencer, C. A., Chu, C. J., Bodien, Y. G., O'Connor, K. L.,... Wu, O. (2017). Early detection of consciousness in patients with acute severe traumatic brain injury. Brain, 140(9), 2399-2414. doi:10.1093/brain/awx176

Formisano, R., Vinicola, V., Penta, F., Matteis, M., Brunelli, S., \& Weckel, J. W. (2001). Active music therapy in the rehabilitation of severe brain injured patients during coma recovery. Ann Ist Super Sanita, 37(4), 627-630.

Frazzitta, G., Zivi, I., Valsecchi, R., Bonini, S., Maffia, S., Molatore, K.,... \& Saltuari, L. (2016). Effectiveness of a Very Early Stepping Verticalization Protocol in Severe Acquired Brain Injured Patients: A Randomized Pilot Study in ICU. PLoS One, 11(7), e0158030. doi:10.1371/journal.pone.0158030

Gelinas, C., Fortier, M., Viens, C., Fillion, L., \& Puntillo, K. (2004). Pain assessment and management in critically ill intubated patients: A retrospective study. Am J Crit Care, 13(2), 126-135.

Gesler, W. M. (1992). Therapeutic landscapes: Medical issues in light of the new cultural geography. Soc Sci Med, 34(7), 735746.

Giacino, J. T., Ashwal, S., Childs, N., Cranford, R., Jennett, B., Katz, D. I.,... \& Zasler, N. D. (2002). The minimally conscious state: Definition and diagnostic criteria. Neurology, 58(3), 349353.

Goldfine, A. M., Victor, J. D., Conte, M. M., Bardin, J. C., \& Schiff, N. D. (2011). Determination of awareness in patients with severe brain injury using EEG power spectral analysis. Clin Neurophysiol, 122(11), 2157-2168. doi:10.1016/j.clinph.2011.03.022

Jarrott, S. E., \& Gigliotti, C. M. (2010). Comparing responses to horticultural-based and traditional activities in dementia care programs. American Journal of Alzheimer's Disease \& Other Dementias $^{\circledR}, 25(8), 657-665$.

Jonasson, I., Marklund, B., \& Hildingh, C. (2007). Working in a training garden: Experiences of patients with neurological damage. Australian Occupational Therapy Journal, 54(4), 266-272.

Kalmar, K., \& Giacino, J. T. (2005). The JFK Coma Recovery Scale-Revised. Neuropsychol Rehabil, 15(3-4), 454-460. doi:10.1080/09602010443000425

Kaplan, R., \& Kaplan, S. (1989). The experience of nature: A psychological perspective: CUP Archive.

Knebel, J. F., Javitt, D. C., \& Murray, M. M. (2011). Impaired early visual response modulations to spatial information in chronic schizophrenia. Psychiatry Res, 193(3), 168-176. doi:10.1016/j.pscychresns.2011.02.006

Kondziella, D., Friberg, C. K., Frokjaer, V. G., Fabricius, M., \& Moller, K. (2016). Preserved consciousness in vegetative and minimal conscious states: Systematic review and metaanalysis. J Neurol Neurosurg Psychiatry, 87(5), 485-492. doi:10.1136/jnnp-2015-310958

Krewer, C., Luther, M., Koenig, E., \& Muller, F. (2015). Tilt Table Therapies for Patients with Severe Disorders of Consciousness: A Randomized, Controlled Trial. PLoS One, 10(12), e0143180. doi:10.1371/journal.pone.0143180

Lanz, F., Moret, V., Rouiller, E. M., \& Loquet, G. (2013). Multisensory Integration in Non-Human Primates during a Sensory-Motor Task. Front Hum Neurosci, 7, 799. doi:10.3389/fnhum.2013.00799

Laureys, S., Celesia, G. G., Cohadon, F., Lavrijsen, J., LeonCarrion, J., Sannita, W. G.,... European Task Force on Disorders of, C. (2010). Unresponsive wakefulness syndrome: A new name for the vegetative state or apallic syndrome. BMC Med, 8, 68. doi:10.1186/1741-7015-8-68

LeWinn, E. B., \& Dimancescu, M. D. (1978). Environmental deprivation and enrichment in coma. Lancet, 2(8081), 156-157.

Lombardi, F., Taricco, M., De Tanti, A., Telaro, E., \& Liberati, A. (2002). Sensory stimulation of brain-injured individuals in coma or vegetative state: Results of a Cochrane systematic review. Clin Rehabil, 16(5), 464-472. doi:10.1191/0269215502cr519oa

Magee, W. L. (2007). Music as a diagnostic tool in low awareness states: Considering limbic responses. Brain Inj, 21(6), 593 599. doi:10.1080/02699050701426907

Maller, C., Townsend, M., Pryor, A., Brown, P., \& St Leger, L. (2006). Healthy nature healthy people: 'contact with nature' as an upstream health promotion intervention for populations. Health Promot Int, 21(1), 45-54. doi:10.1093/heapro/ dai032

Megha, M., Harpreet, S., \& Nayeem, Z. (2013). Effect of frequency of multimodal coma stimulation on the consciousness levels of traumatic brain injury comatose patients. Brain Inj, 27(5), 570-577. doi:10.3109/02699052.2013.767937 
Mitchell, S., Bradley, V. A., Welch, J. L., \& Britton, P. G. (1990). Coma arousal procedure: A therapeutic intervention in the treatment of head injury. Brain Inj, 4(3), 273-279.

Monti, M. M., Vanhaudenhuyse, A., Coleman, M. R., Boly, M., Pickard, J. D., Tshibanda, L.,... \& Laureys, S. (2010). Willful modulation of brain activity in disorders of consciousness. $N$ Engl J Med, 362(7), 579-589. doi:10.1056/NEJMoa0905370

Namazi, K. H., \& Haynes, S. R. (1994). Sensory stimuli reminiscence for patients with Alzheimer's disease: Relevance and implications. Clinical Gerontologist, 14(4), 29-46.

Oh, H., \& Seo, W. (2003). Sensory stimulation programme to improve recovery in comatose patients. J Clin Nurs, 12(3), 394-404.

Ohly, H., White, M. P., Wheeler, B. W., Bethel, A., Ukoumunne, O. C., Nikolaou, V., \& Garside, R. (2016). Attention Restoration Theory: A systematic review of the attention restoration potential of exposure to natural environments. J Toxicol Environ Health B Crit Rev, 19(7), 305-343. doi:10.1080/10937404.2016.1196155

Owen, A. M., Coleman, M. R., Boly, M., Davis, M. H., Laureys, S., \& Pickard, J. D. (2006). Detecting awareness in the vegetative state. Science, 313(5792), 1402. doi:10.1126/science.1130197

Phelps, E. A. (2006). Emotion and cognition: Insights from studies of the human amygdala. Annu Rev Psychol, 57, 27-53. doi:10.1146/annurev.psych.56.091103.070234

Pignat, J. M., Mauron, E., Johr, J., Gilart de Keranflec'h, C., Van De Ville, D., Preti, M. G.,... \& Diserens, K. (2016). Outcome Prediction of Consciousness Disorders in the Acute Stage Based on a Complementary Motor Behavioural Tool. PLoS One, 11(6), e0156882. doi:10.1371/journal.pone.0156882

Pincherle, A., Jöhr, J., Chatelle, C., Pignat, J. M., Du Pasquier, R., Ryvlin, P., Oddo, M. \& Diserens, K. (2019). Motor behavior unmasks residual cognition in disorders of consciousness. Ann Neurol, 85(3), 443-447. doi:10.1002/ana.25417

Plum, F., \& Posner, J. B. (1972). The diagnosis of stupor and coma. Contemp Neurol Ser, 10, 1-286.

Schiff, N. D. (2015). Cognitive Motor Dissociation Following Severe Brain Injuries. JAMA Neurol, 72(12), 1413-1415. doi:10.1001/jamaneurol.2015.2899
Schnakers, C., Perrin, F., Schabus, M., Majerus, S., Ledoux, D., Damas, P.,... \& Laureys, S. (2008). Voluntary brain processing in disorders of consciousness. Neurology, 71(20), 1614-1620. doi:10.1212/01.wnl.0000334754.15330.69

Spiegel, D. R., Casella, D. P., Callender, D. M., \& Dhadwal, N. (2008). Treatment of akinetic mutism with intramuscular olanzapine: A case series. J Neuropsychiatry Clin Neurosci, 20(1), 93-95. doi:10.1176/jnp.2008.20.1.93

Taub, E., Crago, J. E., Burgio, L. D., Groomes, T. E., Cook III, E. W., DeLuca, S. C., \& Miller, N. E. (1994). An operant approach to rehabilitation medicine: Overcoming learned nonuse by shaping. Journal of the Experimental Analysis of Behavior, 61(2), 281-293.

Ulrich, R. S. (1984). View through a window may influence recovery from surgery. Science, 224(4647), 420-421.

Ulrich, R. S., Zimring, C., Zhu, X., DuBose, J., Seo, H.-B., Choi, Y.-S.,... Joseph, A. (2008). A review of the research literature on evidence-based healthcare design. HERD: Health Environments Research \& Design Journal, 1(3), 61-125.

Vuilleumier, P. (2005). How brains beware: Neural mechanisms of emotional attention. Trends Cogn Sci, 9(12), 585-594. doi:10.1016/j.tics.2005.10.011

Währborg, P., Petersson, I. F., \& Grahn, P. (2014). Nature-assisted rehabilitation for reactions to severe stress and/or depression in a rehabilitation garden: Long-term follow-up including comparisons with a matched population-based reference cohort. Journal of Rehabilitation Medicine, 46(3), 271-276.

Walch, J. M., Rabin, B. S., Day, R., Williams, J. N., Choi, K., \& Kang, J. D. (2005). The effect of sunlight on postoperative analgesic medication use: A prospective study of patients undergoing spinal surgery. Psychosomatic Medicine, 67(1), 156-163.

Wilson, E. O. (1984). Biophilia. Harvard. Press, Cambridge (Mass.).

Yarns, B. C., \& Quinn, D. K. (2013). Telephone effect in akinetic mutism from traumatic brain injury. Psychosomatics, 54(6), 609-610. doi:10.1016/j.psym.2013.06.006 